\title{
An exact solution of torsional quartz crystal resonators of free-free bar-type
}

\author{
Hirofumi Kawashima and Kenji Sunaga \\ Seiko Instruments Inc., \\ 563, Takatsuka-shinden, Matsudo, 271 Japan
}

(Received 26 October 1995)

\begin{abstract}
An exact solution of a partial differential equation including elastic compliance constant $s_{56}{ }^{\prime}$, with respect to stress function $\Psi$ has been found for torsional modes of vibration of an arbitrary (singly, doubly, triply) rotated quartz crystal beam with a pair of parallel, free edges. The solution is obtained by relaxing the condition that the edge planes are perpendicular to the main faces of the beam. That is, the edges are off perpendicular by the angle $\Theta=\arctan \left(-s_{56}{ }^{\prime} / s_{55}{ }^{\prime}\right)$. The exact solution can reduce the difference of the calculated and measured values of frequency constant for a thickness-to-width ratio. Also, a comparatively large-inclination of the edge cuts is required to reduce the unwanted, complicated mode shapes to simple ones.
\end{abstract}

Keywords: Torsional vibration, Piezo-element, Supersonic wave, Free vibration, Exact solution, Quartz crystal, Frequency constant

PACS number: 43. 88. $-\mathrm{p}$

\section{INTRODUCTION}

Since a frequency constant of a resonator vibrating in torsion ${ }^{1-3)}$ lies intermediately to those for flexural and length extensional mode resonators, it is very available for realization of miniature resonators of a comparatively low-frequency, i.e., 400 to $600 \mathrm{kHz}$. In order that one of the authors clarifies frequency temperature behavior of doubly rotated torsional quartz crystal resonators of tuning fork-type which can be approximately regarded as a clamped-free bar, and free-free bar-type, a theoretical and/or experimental examination was performed in detail. As a result, we have already obtained and reported ${ }^{1,2)}$ that a cut angle $(\phi, \theta)$ where gives the first order temperature coefficient $\alpha=0$ and approximately one third of the second order temperature coefficient $\beta$ for flexural mode quartz crystal resonators, exists for the torsional quartz crystal resonator by combination of the cut angle $(\phi, \theta)$ and a thickness-to-width ratio, the so called TT-Cut classified into the TT $\left(\mathrm{X}_{1}\right)$ - and
$\mathrm{TT}\left(\mathrm{X}_{2}\right)$-Cuts.

A vibration problem of torsion is equivalent to solving the problem of torsional rigidity $C_{\mathrm{t}}$. In the previous report ${ }^{2)} C_{\mathrm{t}}$ was derived from stress function $\Psi$. However, the obtained stress function $\Psi$ is an approximate solution derived neglecting the elastic compliance constant which couples two shear stresses.

In this paper, an exact solution of torsional rigidity $C_{\mathrm{t}}$ for a torsional mode resonator is found and applied to quartz crystal. In analysis procedure, first, a frequency equation is, as already reported, ${ }^{1-3)}$ found to be given as a function of torsional rigidity $C_{\mathrm{t}}$. Next, a general strain-stress relation is transformed into a simple one from the conditions of resonator shape and dimensions, and from the equation of equilibrium a partial differential equation is derived with respect to stress function $\Psi$. In addition, the $\Psi$ which satisfies the boundary conditions is derived and torsional rigidity $C_{\mathrm{t}}$ is calculated. Finally, the obtained result is applied to a quartz crystal resonator of free-free bar-type and is com- 
pared with the measured ones of the quartz crystal resonator vibrating in torsion. Consequently, the calculated values taking account of $s_{56}{ }^{\prime}$ are found to agree with the measured ones better than those neglecting $s_{56}{ }^{\prime}$.

\section{ANALYSIS PROCEDURE}

\subsection{Frequency Equation}

Figure 1 shows a resonator of a beam vibrating in torsion and its coordinate system. The resonator is expressed in the IEEE notation of (YXlt) $\phi / \theta$ and has a dimension of length $2 x_{0}$, width $2 z_{0}$ and thickness $2 y_{0}$. When length $2 x_{0}$ is much larger than width $2 z_{0}$ and thickness $2 y_{0}$, lateral motion for a resonator vibrating in torsion can be neglected. ${ }^{4)}$ A resonant frequency $f$ for a torsional resonator is, therefore, given as

$$
f=\frac{m}{4\left(2 x_{0}\right)} \sqrt{\frac{C_{\mathrm{t}}}{\rho I_{0}}},
$$

where

$C_{\mathrm{t}}:$ torsional rigidity

$\rho:$ density of beam

$I_{0}$ : polar moment of inertia

$2 x_{0}$ : length of beam

$m$ : even for free-free beam and odd for clamped-free beam.

As can be easily understood from Eq. (1), a vibration problem of torsion is equivalent to solving the problem of torsional rigidity $C_{\mathrm{t}}$ because $I_{0}$ is determined by the resonator shape of section.

\subsection{Equations of Equilibrium}

Now, when displacements in the $x, y$ and $z$ axes direction are taken as $u, v$ and $w$, the displacements $u, v, w$ can be expressed by

$$
\begin{aligned}
& u=\tau \Phi(y, z) \\
& v=-\tau x z \\
& w=\tau x y,
\end{aligned}
$$

where torsion function $\Phi$ is a function of $y$ and $z$,

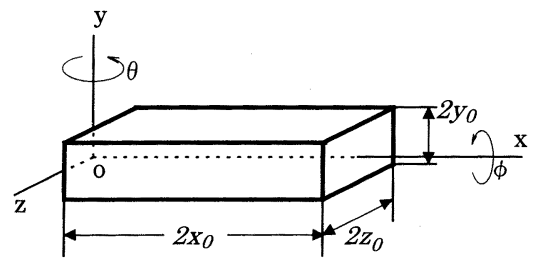

Fig. 1 Torsional resonator of a beam and its coordinate system. and $\tau$ is the twist. From the relation of Eq. (2), the strain-components vanish and are

$$
S_{1}=S_{2}=S_{3}=S_{4}=0 .
$$

A general strain-stress relation has the form

$$
\begin{aligned}
& S_{1}=s_{11} T_{1}+s_{12} T_{2}+s_{13} T_{3}+s_{14} T_{4}+s_{15} T_{5}+s_{16} T_{6} \\
& S_{2}=s_{21} T_{1}+s_{22} T_{2}+s_{23} T_{3}+s_{24} T_{4}+s_{25} T_{5}+s_{26} T_{6} \\
& S_{3}=s_{31} T_{1}+s_{32} T_{2}+s_{33} T_{3}+s_{34} T_{4}+s_{35} T_{5}+s_{36} T_{6} \\
& S_{4}=s_{41} T_{1}+s_{42} T_{2}+s_{43} T_{3}+s_{44} T_{4}+s_{45} T_{5}+s_{46} T_{6} \\
& S_{5}=s_{51} T_{1}+s_{52} T_{2}+s_{53} T_{3}+s_{54} T_{4}+s_{55} T_{5}+s_{56} T_{6} \\
& S_{6}=s_{61} T_{1}+s_{62} T_{2}+s_{63} T_{3}+s_{64} T_{4}+s_{65} T_{5}+s_{66} T_{6},
\end{aligned}
$$

where $s_{q p}$ is elastic compliance constant. From Eq. (3) the strain-stress relation reduces to

$$
\begin{aligned}
& S_{5}={ }_{S_{55}}{ }^{\prime} T_{5}+{ }_{S_{56}}{ }^{\prime} T_{6} \\
& S_{6}=S_{65}{ }^{\prime} T_{5}+{ }_{S_{66}}{ }^{\prime} T_{6},
\end{aligned}
$$

where

$$
\begin{aligned}
& S_{q p}{ }^{\prime}=\widehat{s}_{q p}-\widehat{s}_{4 q} \widehat{S}_{p 4} / \widehat{S}_{44} \quad(q, p=5,6) \\
& \widehat{s}_{q p}=\tilde{s}_{q p}-\tilde{s}_{3 q} \widetilde{s}_{p 3} / \widetilde{s}_{33} \quad(q, p=4,5,6) \\
& \tilde{s}_{q p}=\bar{s}_{q p}-\bar{s}_{2 q} \bar{s}_{p 2} / \bar{s}_{22} \quad(q, p=3,4,5,6) \\
& \bar{s}_{q p}=s_{q p}-s_{1 q} s_{p 1} / s_{11} \quad(q, p=2,3,4,5,6) .
\end{aligned}
$$

In addition, the equations of equilibrium yield

$$
\begin{aligned}
& \frac{\partial T_{1}}{\partial x}+\frac{\partial T_{6}}{\partial y}+\frac{\partial T_{5}}{\partial z}=0 \\
& \frac{\partial T_{6}}{\partial x}+\frac{\partial T_{2}}{\partial y}+\frac{\partial T_{4}}{\partial z}=0 \\
& \frac{\partial T_{5}}{\partial x}+\frac{\partial T_{4}}{\partial y}+\frac{\partial T_{3}}{\partial z}=0 .
\end{aligned}
$$

Since stress-components $T_{1}, T_{5}$ and $T_{6}$ are given as a function of $y$ and $z$, we find that

$$
\frac{\partial T_{1}}{\partial x}=\frac{\partial T_{5}}{\partial x}=\frac{\partial T_{6}}{\partial x}=0 .
$$

When length $2 x_{0}$ for a torsional resonator is much larger than width $2 z_{0}$ and thickness $2 y_{0}$, stresscomponents $T_{2}, T_{3}$ and $T_{4}$ become

$$
T_{2}=T_{3}=T_{4}=0 .
$$

From Eqs. (7) and (8), the second and third equations of Eq. (6) are always satisfied, so that the equation of equilibrium is given by

$$
\frac{\partial T_{6}}{\partial y}+\frac{\partial T_{5}}{\partial z}=0 \text {. }
$$

Equation (9) is satisfied if

$$
T_{5}=-\frac{\partial \Psi}{\partial y} \text { and } T_{6}=\frac{\partial \Psi}{\partial z},
$$

$\Psi$ is stress function. Substitution of Eqs. (2) and (10) into Eq. (5) then yields 


$$
\begin{gathered}
S_{55} \frac{\partial^{2} \Psi}{\partial y^{2}}+S_{66}{ }^{\prime} \frac{\partial^{2} \Psi}{\partial z^{2}}-2 S_{56} \frac{\partial^{2} \Psi}{\partial y \partial z}=-2 \tau, \\
\left(\because \quad S_{56}{ }^{\prime}=S_{65}{ }^{\prime}\right)
\end{gathered}
$$

which must hold over the area of the cross section, while the boundary conditions must be satisfied with

$$
\begin{array}{lll}
T_{5}=0 & \text { on } & z= \pm z_{0} \\
T_{6}=0 & \text { on } & y= \pm y_{0} .
\end{array}
$$

Hence, the stress function $\Psi$ is derived from Eq. (11) and the boundary conditions of Eq. (12).

\subsection{Stress Function $\Psi$}

Now, we take the stress function $\Psi$ as

$$
\Psi=a_{n}\left\{1-b_{n} \cosh \xi(z+\zeta y)\right\} \cos \eta y .
$$

where $a_{n}, b_{n}, \xi, \xi$ and $\eta$ are constant. Substitution of Eq. (13) into Eq. (11) then gives

$$
\begin{aligned}
& a_{n}\left\{b _ { n } \left(s_{55}{ }^{\prime} \eta^{2}-s_{55}{ }^{\prime} \xi^{2} \zeta^{2}-s_{66}{ }^{\prime} \xi^{2}\right.\right. \\
& \left.\quad+2 s_{56}{ }^{\prime} \xi^{2} \zeta\right) \cosh \xi(z+\zeta y) \cos \eta y \\
& \quad+2 b_{n}\left(s_{55} \eta \xi \xi-s_{56}{ }^{\prime} \eta \xi\right) \sinh \xi(z+\zeta y) \\
& \left.\quad \cdot \sin \eta y-\eta^{2} s_{55}{ }^{\prime} \cos \eta y\right\}=-2 \tau .
\end{aligned}
$$

Accordingly, to hold Eq. (14) at an arbitrary value of $\cosh \xi(z+\xi y) \cos \eta y$ and $\sinh \xi(z+\xi y) \sin \eta y$, we should find that

$$
\begin{aligned}
& s_{55}{ }^{\prime} \eta^{2}-S_{55}{ }^{\prime} \xi^{2} \zeta^{2}-s_{66}{ }^{\prime} \xi^{2}+2 S_{56}{ }^{\prime} \xi^{2} \zeta=0 \\
& s_{55}{ }^{\prime} \eta \xi \zeta-s_{56} \eta \xi=0 .
\end{aligned}
$$

From Eq. (15) $\xi$ and $\xi$, respectively, become

$$
\begin{aligned}
& \zeta=\frac{S_{56}{ }^{\prime}}{S_{55}{ }^{\prime}} \\
& \xi=\sqrt{\frac{S_{55^{\prime}}}{S_{66}{ }^{\prime}}} \frac{1}{\sqrt{1-\frac{S_{56^{\prime}{ }^{\prime}}}{S_{55} S_{66}{ }^{\prime}}}} \eta
\end{aligned}
$$

so that Eq. (14) is simplified into

$$
a_{n} \eta^{2} S_{55}{ }^{\prime} \cos \eta y=2 \tau \text {. }
$$

Now when $-y_{0}<y<+y_{0}$, the $2 \tau$ of the right term in Eq. (18) can be expanded in a Fourier's series ${ }^{5}$ as follows :

$$
2 \tau=2 \tau \sum_{n=1,3,5}^{m} \frac{4}{n \pi}(-1)^{(n-1) / 2} \cos \eta y .
$$

Hence, $a_{n}$ of Eq. (18) can be expressed in the form

$$
a_{n}=\frac{8 \tau}{\eta^{2} \pi} \frac{1}{S_{55}{ }^{\prime}} \sum_{n=1,3,5}^{m} \frac{1}{n}(-1)^{(n-1) / 2} .
$$

Next, the constants $\eta$ and $b_{n}$ are derived from the boundary conditions. The stress-component $T_{6}$ of Eq. (10) becomes

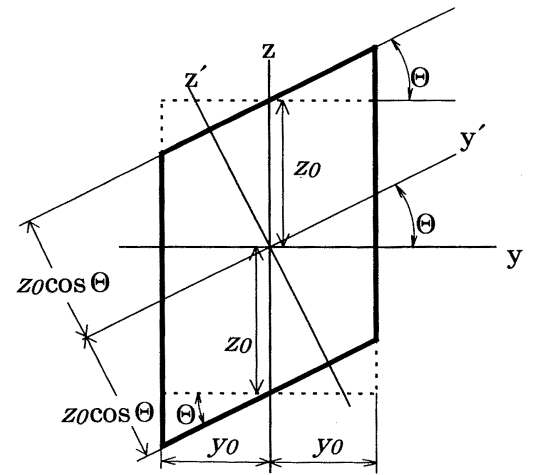

Fig. 2 Sectional view and rotated axes of quartz crystal resonator.

$$
\begin{aligned}
T_{6} & =\frac{\partial \Psi}{\partial z} \\
& =a_{n} b_{n} \xi \sinh \xi(z+\zeta y) \cos \eta y,
\end{aligned}
$$

the boundary condition of $T_{6}=0$ on $y= \pm y_{0}$ then gives

$$
\eta=\frac{n \pi}{2 y_{0}} \quad(n=1,3,5, \cdots) .
$$

In addition, since we can not find a solution which satisfies the boundary condition of $T_{5}=0$ on $z=$ $\pm z_{0}$, the edges are, as illustrated in Fig. 2, off perpendicular by the angle $\Theta$. When a resonator vibrating in torsion is rotated about the $x$-axis, a transformation of an old coordinate system $(x, y, z)$ into a new one $\left(x^{\prime}, y^{\prime}, z^{\prime}\right)$ yields

$$
\left(\begin{array}{l}
x^{\prime} \\
y^{\prime} \\
z^{\prime}
\end{array}\right)=\left(\begin{array}{ccc}
1 & 0 & 0 \\
0 & \cos \Theta & \sin \Theta \\
0 & -\sin \Theta & \cos \Theta
\end{array}\right)\left(\begin{array}{l}
x \\
y \\
z
\end{array}\right) .
$$

Accordingly, the transformed stress-components $T_{5}{ }^{\prime}$ and $T_{6}^{\prime}$ is given by

$$
\begin{aligned}
& T_{5}^{\prime}=T_{5} \cos \Theta-T_{6} \sin \Theta \\
& T_{6}^{\prime}=T_{5} \sin \Theta+T_{6} \cos \Theta .
\end{aligned}
$$

In addition, the stress-components $T_{5}$ and $T_{6}$ are transformed into

$$
\begin{aligned}
T_{5}=-\frac{\partial \Psi}{\partial y}= & a_{n}\left[b_{n} \xi \zeta \sinh \xi(z+\zeta y) \cos \eta y\right. \\
& \left.+\eta \sin \eta y\left\{1-b_{n} \cosh \xi(z+\zeta y)\right\}\right] \\
T_{6}=\frac{\partial \Psi}{\partial z}=- & a_{n} b_{n} \xi \sinh \xi(z+\zeta y) \cos \eta y
\end{aligned}
$$

From the relation of Eq. (25) $T_{5}^{\prime}$ reduces to

$$
\begin{aligned}
T_{5}^{\prime}= & a_{n}\left[b_{n} \xi(\zeta \cos \Theta+\sin \Theta) \sinh \xi(z+\zeta y) \cos \eta y\right. \\
& \left.+\eta \cos \Theta \sin \eta y\left\{1-b_{n} \cosh \xi(z+\zeta y)\right\}\right]
\end{aligned}
$$

In Eq. (26), we set 


$$
\zeta \cos \Theta+\sin \Theta=0,
$$

so that we obtain

$$
\Theta=\arctan \left(-S_{56}{ }^{\prime} / S_{55}{ }^{\prime}\right) .
$$

Here we note that $z+\xi y=z^{\prime} / \cos \Theta, T_{5}^{\prime}$ reduces to

$$
T_{5}^{\prime}=a_{n} \eta \cos \Theta \sin \eta y\left(1-b_{n} \cosh \frac{\xi}{\cos \Theta} z^{\prime}\right) .
$$

From the boundary condition of $T_{5}^{\prime}=0$ on $z^{\prime}=$ $\pm z_{0} \cos \Theta$, we should find that

$$
b_{n}=\frac{1}{\cosh \xi z_{0}} .
$$

From these results, stress function $\Psi$ is given by

$$
\begin{aligned}
\Psi= & \frac{8 \tau\left(2 y_{0}\right)^{2}}{\pi^{3}} \frac{1}{S_{55}{ }^{\prime}} \sum_{n=1,3,5}^{m} \frac{1}{n^{3}}(-1)^{(n-1) / 2} \\
& \times\left[1-\frac{\cosh \xi(z+\zeta y)}{\cosh \xi z_{0}}\right] \cos \eta y .
\end{aligned}
$$

Namely, $\Psi$ of Eq. (31) is an exact solution which includes the elastic compliance constant $s_{56}{ }^{\prime}$.

\subsection{Torsional Rigidity $C_{\mathrm{t}}$}

Since torsional rigidity $C_{\mathrm{t}}$ is given by a ratio of torsional moment $M_{\mathrm{t}}$ and the twist $\tau\left(M_{\mathrm{t}} / \tau\right)$, the $M_{\mathrm{t}}$ which is defined by the area integration of $\Psi$, i.e., $2 \int_{-z_{0}}^{z_{0}} \int_{-y_{0}}^{y_{0}} \Psi d y d z$ is calculated, so that $M_{\mathrm{t}}$ is

$$
\begin{aligned}
& M_{\mathrm{t}}=\frac{1}{3} \tau \frac{1}{S_{55}{ }^{\prime}}\left(2 y_{0}\right)^{3}\left(2 z_{0}\right)\left[1-\frac{192}{\pi^{5}}\left(\frac{y_{0}}{z_{0}}\right)\right. \\
& \times \sqrt{\frac{S_{66}{ }^{\prime}}{S_{55}{ }^{\prime}}} \sqrt{1-\frac{S_{56}{ }^{\prime 2}}{S_{55}{ }^{\prime} S_{66}{ }^{\prime}}}\left(1-\frac{S_{56}{ }^{\prime 2}}{S_{55}{ }^{\prime} S_{66}{ }^{\prime}}\right) \\
& \times \sum_{n=1,3,5}^{m} \frac{1}{n^{5}} \cosh \left(\frac{n \pi}{2} \frac{S_{56^{\prime}}}{\sqrt{S_{55} S_{66}^{\prime}}} \frac{1}{\sqrt{1-\frac{S_{56^{\prime}{ }^{\prime 2}} S_{55} S_{66}{ }^{\prime}}{}}}\right) \\
& \times \tanh \left(\frac{n \pi}{2} \sqrt{\frac{S_{55}{ }^{\prime}}{S_{66}^{\prime}}} \frac{1}{\sqrt{1-\frac{S_{56}{ }^{\prime 2}}{S_{55} S_{66}^{\prime}}}} \frac{z_{0}}{y_{0}}\right) \text {. }
\end{aligned}
$$

Here, we note that the constant $m$ must be so determined that it fulfills the convergent condition of $M_{\mathrm{t}}$. Namely, it may need the following relation

$$
\sqrt{s_{55}{ }^{\prime} S_{66}{ }^{\prime}-s_{56}{ }^{\prime 2}}>m s_{56}{ }^{\prime},
$$

From the relation of Eq. (32) torsional rigidity $C_{\mathrm{t}}$ is given by

$$
\begin{aligned}
C_{\mathrm{t}}= & \frac{1}{3} \frac{1}{S_{55}{ }^{\prime}}\left(2 y_{0}\right)^{3}\left(2 z_{0}\right)\left[1-\frac{192}{\pi^{5}}\left(\frac{y_{0}}{z_{0}}\right)\right. \\
& \cdot \sqrt{\frac{S_{66}{ }^{\prime}}{S_{55}{ }^{\prime}}} \sqrt{1-\frac{S_{56}{ }^{\prime 2}}{S_{55} S_{66}{ }^{\prime}}}\left(1-\frac{S_{56^{\prime}}{ }^{\prime 2}}{S_{55} S_{66}^{\prime}}\right)
\end{aligned}
$$

$$
\begin{gathered}
\times \sum_{n=1,3.5}^{m} \frac{1}{n^{5}} \cosh \left(\frac{n \pi}{2} \frac{S_{56^{\prime}}}{\sqrt{S_{55}{ }^{\prime} S_{66}{ }^{\prime}}} \frac{1}{\sqrt{1-\frac{S_{56}{ }^{\prime 2}}{S_{55}{ }^{\prime} S_{66}{ }^{\prime}}}}\right) \\
\left.\cdot \tanh \left(\frac{n \pi}{2} \sqrt{\frac{S_{55}{ }^{\prime}}{S_{66}{ }^{\prime}}} \frac{1}{\sqrt{1-\frac{S_{56}{ }^{\prime 2}}{S_{55}{ }^{\prime} S_{66}{ }^{\prime}}}} \frac{z_{0}}{y_{0}}\right)\right] .
\end{gathered}
$$

In addition, when $s_{56}{ }^{\prime}=0, C_{\mathrm{t}}$ reduces to

$$
\begin{aligned}
C_{\mathrm{t}}= & \frac{1}{3} \frac{1}{S_{55}{ }^{\prime}}\left(2 y_{0}\right)^{3}\left(2 z_{0}\right)\left[1-\frac{192}{\pi^{5}}\left(\frac{y_{0}}{z_{0}}\right) \sqrt{\frac{S_{66}{ }^{\prime}}{S_{55}{ }^{\prime}}}\right. \\
& \left.\times \sum_{n=1,3,5}^{\infty} \frac{1}{n^{5}} \tanh \left(\frac{n \pi}{2} \sqrt{\frac{S_{55}{ }^{\prime}}{S_{66}{ }^{\prime}} y_{0}}\right)\right],
\end{aligned}
$$

this result agrees with those ${ }^{1,2,5,6)}$ derived from the first condition of $s_{56}{ }^{\prime}=0$.

\section{RESONATOR SHAPE AND EXCITATION ELECTRODES}

Figure 3 shows resonator shape and dimensions of free-free bar-type vibrating in torsion which are used in the present experiments (a), and the sectional view of electrodes construction (b). The resonator has a dimension of $x_{1}=0.8 \mathrm{~mm}, x_{2}=1.66 \mathrm{~mm}, x_{3}=$ $80 \mu \mathrm{m}, z_{1}=2 z_{0}+2 z_{2}+2 z_{3}, z_{2}=200 \mu \mathrm{m}, z_{3}=100 \mu \mathrm{m}$ and $2 y_{0}=0.12 \mathrm{~mm}$, and are fabricated by a chemical etching process. The edges of arms of the beam are off perpendicular by the angle $\Theta$. When the resonator has a cut angle of $\phi=66^{\circ}, \theta=0^{\circ}$ and $\Theta=$ $24^{\circ}$, an error for $\Theta=24^{\circ}$ which is caused by masking accuracy and etching technology is within $\pm 40^{\prime}$ according to measurement of $X$ rays. The resonators are formed from a rotated Y-plate with a cut angle of $\phi$ and $\theta$ about the $x$ - and $y^{\prime}$-axes. In addition, the excitation electrodes are deposited on

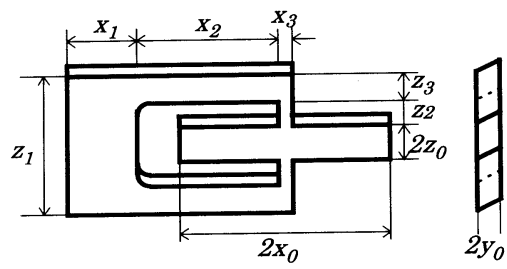

(a)

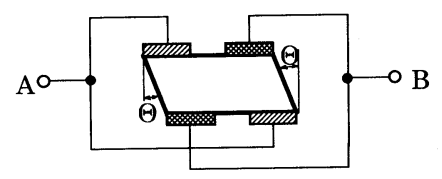

(b)

Fig. 3 Shape and dimensions of torsional quartz crystal resonator of free-free bar-type (a) and its sectional view of electrodes construction (b). 


\section{H. KAWASHIMA and K. SUNAGA : AN EXACT SOLUTION OF TORSIONAL QCRS FREE-FREE BAR}

obverse and reverse of the beam and the adjoining electrodes have different polarity.

\section{RESULTS and DISCUSSION}

The results obtained in the present analysis are compared with the measured values of torsional quartz crystal resonators of free-free bar-type. Namely, a relationship of a frequency constant versus a thickness-to-width ratio $R_{y z}$ is shown. In the calculation the constants of the Ref. 7) are employed.

Figure 4 shows the relationship of a frequency constant $\left(f \cdot 2 x_{0}\right)$ to a thickness-to-width ratio $R_{y z}$ of a quartz crystal resonator vibrating in torsion with a cut angle of $\phi=66^{\circ}$ and $\theta=0^{\circ}$, and an edge angle of $\Theta=24^{\circ}$. The solid and broken lines are the calculated values when $S_{56}$ is taken and neglected, respectively, and circles are the measured ones. As is apparent from Fig. 4, the frequency constant $(f$. $2 x_{0}$ ) analyzed taking $s_{56}{ }^{\prime}$ into account becomes larger than that analyzed neglecting $S_{56}{ }^{\prime}$, particularly, in the range of $R_{y z}=0.5$ to a crossing of $R_{y z}=$ 1.2 approximately. The calculated values taking account of $s_{56}{ }^{\prime}$ agree with the measured ones better than those neglecting $S_{56}{ }^{\prime}$. The frequency constant $\left(f \cdot 2 x_{0}\right)$ is approximately proportional to the aspect ratio $R_{y z}$ when it is small. As is shown in Fig. 4, the frequency constant $\left(f \cdot 2 x_{0}\right)$ increases gradually according to increase of the aspect ratio $R_{y z}$. However, further increase of $R_{y z}$ gives the maximum of the frequency constant in the vicinity of $R_{y z}=0.9$, and then, an inclination to decrease gradually. For example, the frequency constant $\left(f \cdot 2 x_{0}\right)$ is 182.5 $\mathrm{kHz} \cdot \mathrm{cm}$ in the calculation and $181.2 \mathrm{kHz} \cdot \mathrm{cm}$ in the experiments when a thickness-to-width ratio $R_{y z}$ is 1.017. Since these values lie intermediately to those for flexural and length extensional mode resonators,

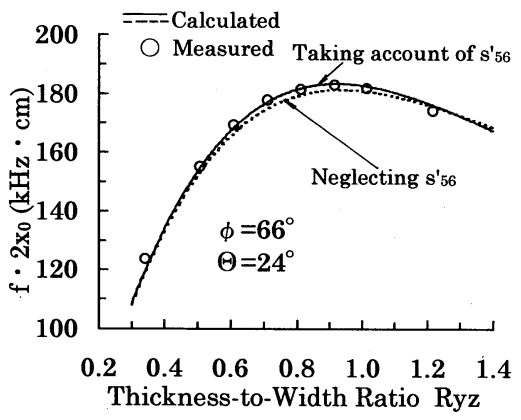

Fig. 4 Relationship of frequency constant $(f$. $\left.2 x_{0}\right)$ and thickness-to-width ratio $R_{y z}$. a miniature torsional quartz crystal resonator of 400 to $600 \mathrm{kHz}$ can be realized when the resonator size is taken into account.

\section{CONCLUSIONS}

In this paper, an exact solution of torsional rigidity $C_{\mathrm{t}}$ for a resonator vibrating in torsion has been found. This theoretical result was applied to torsional quartz crystal resonators of free-free bar-type and the calculated values are compared with the measured ones.

First, the general strain-stress relation was transformed into a simple one from the conditions of resonator shape and dimensions, and from the equation of equilibrium the partial differential equation was derived with respect to the stress-function $\Psi$. In addition, the $\Psi$ which satisfies the boundary conditions was derived and then, the torsional rigidity $C_{\mathrm{t}}$ was calculated.

Second, the relationship of a frequency constant $\left(f \cdot 2 x_{0}\right)$ versus a thickness-to-width ratio $R_{y z}$ was clarified from the theoretically obtained equation, so that the calculated values were found to agree well with the measured ones, e.g., in the vicinity of the aspect ratio $R_{y z}=1.0$.

For the coming subject the influence of the piezoelectric terms on resonant frequency will be clarified.

\section{REFERENCES}

1) H. Kawashima and M. Nakazato, "A new cut torsional quartz crystal resonator of tuning fork-type," Trans. IEICE (A) J76-A, No. 1, pp. 1-8, 1993 (in Japanese).

2) H. Kawashima and M. Nakazato, "TT- cut torsional quartz crystal resonators of free-free bar-type,” Jpn. J. Appl. Phys. 33 Pt. 1, No. 5B, 2989-2993 (1994).

3) J. Hermann, "DT-cut torsional resonator," Proc. 31st Annu. Symp. Freq. Control, U.S.A., 55-61 (1977).

4) H. Kawashima and K. Sunaga, "A vibration analysis of torsional quartz crystal resonators taking account of lateral motion,” Jpn. J. Appl. Phys. 34 Pt. 1, No. 5B, 3316-3319 (1995).

5) A.E.H. Love, $A$ Treatise on the Mathematical Theory of Elasticity, 4th ed. (Cambridge University Press, Cambridge, England, 1952).

6) H. Kawashima, M. Nakazato, and Y. Shimizu, "Quartz temperature sensors of torsional vibration formed from doubly rotated plates," Jpn. J. Appl. Phys. 33, Pt. 1, No.5B, 3005-3009 (1994).

7) M. Aruga, "On the elastic constants of quartz and their temperature characteristics," Bull. Tokyo Inst. Tech. A-2, 88-182 (1956) (in Japanese). 


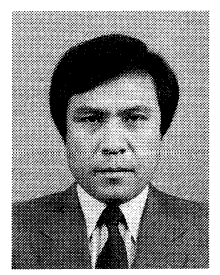

Hirofumi Kawashima was born in Gunma, Japan. He received the B.S. degree in physics from Science University of Tokyo in 1970 and the Ph.D. degree in electrical engineering from Waseda University in 1986. In 1971, he joined Daini Seiko-sha (presently Seiko Instruments, Inc.) Tokyo, Japan. Since then, he has been engaged in the research and development of quartz crystal resonators for wristwatches, NSGT cut coupling quartz crystal resonators, face shear mode quartz crystal renonators, length-extensional mode quartz crystal resonators, quartz crystal sensors (temperature, vacuum, weight, acceleration, thin film), high-precision quartz crystal oscillators (S-TCXO, VC-S-TCXO), and piezoelectric actuators. $\mathrm{He}$ has published more than 50 technical papers, and holds over 30 patents, and numerous foreign patents. Dr. Kawashima is the Lecturer for Yamanashi University for 1994-1995. He has also received numerous awards including: The Minister Award of Science and Technology from the Science and Technology Agency of Japan in 1993, the 1993 IEEE Cady Award in 1993 and the Okochi Memorial Prize from Okochi Memorial Foundation of Japan in 1995. He is a coauthor of Manufacture and Applications of Piezoelectric Materials. He is a senior member of the Institute of Electrical and Electronics Engineers, the Japan Society of Applied Physics, and so on.

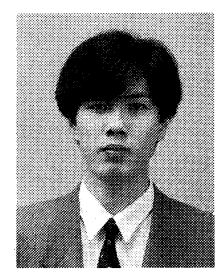

Kenji Sunaga was born in Gunma, Japan on February 12, 1965 . He graduated from Engineering College of Gunma in 1986. In 1986, he joined Seiko Electronics Components (presently Seiko Instruments, Inc.) Tochigi, Japan. Since then, he has been engaged in the research and development of quartz crystal resonators and high-precision quartz crystal oscillators (S-TCXO). Mr. Sunaga received the Aoki Award from the Horological Institute of Japan in 1994. He is a member of the Horological Institute of Japan, the Acoustical Society of Japan and the Institute of Electronic, Information and Communication Engineers. 